Proc. 15th Int. Conference on Defects Recognition, Imaging and Physics in Semiconductors, Warsaw, Poland 2013

\title{
X-Ray Photoelectron Spectroscopy — Methodology and Application
}

\author{
E. Papis-Polakowska ${ }^{a *}$, R.G. White ${ }^{b}$, C. Deeks $^{b}$, M. Mannsberger $^{b}$, A. Krajewska $^{c}$, \\ W. Strupinski ${ }^{c}$, T. PŁOCINSKI ${ }^{d}$ AND O. JANKOWskA ${ }^{e}$ \\ ${ }^{a}$ Institute of Electron Technology, al. Lotników 32/46, 02-668 Warszawa, Poland \\ ${ }^{b}$ Thermo Fisher Scientific, 81 Wyman St., Waltham (MA) 02454 USA \\ ${ }^{c}$ Institute of Electronic Materials Technology, Wólczyńska 133, 01-919 Warszawa, Poland \\ ${ }^{d}$ Warszawa University of Technology, Faculty of Materials Science and Engineering \\ Wołoska 141, 02-507 Warszawa, Poland \\ ${ }^{e}$ COMEF, Gdańska 2, 40-719 Katowice, Poland
}

\begin{abstract}
The crucial measurements aspects of X-ray photoelectron spectroscopy, such as chemical state analysis, depth profiling, mapping, and thickness calculation have been presented. The metal alloys, $\mathrm{Ti}_{2} \mathrm{O}_{5}$, graphene and type-II InAs/GaSb superlattice structures have been examined by using the new Thermo Scientific K-Alpha X-ray Photoelectron Spectrometer.
\end{abstract}

DOI: $10.12693 /$ APhysPolA.125.1061

PACS: 79.60.-i, 81.05.ue, 68.47.Gh, 68.35.bg, 68.35.bj

\section{Introduction}

The chemical composition of surface and interface determines many properties of materials. X-ray photoelectron spectroscopy (XPS) is a well known non-destructive, surface sensitive and quantitative analytical technique used in the surface study. XPS provides information about the elemental composition, empirical formula of pure material, surface contamination, and chemical/electronic state of elements. Especially, the uniformity of elemental composition across the top surface (line profiling or mapping) and uniformity of elemental composition as a function of ion beam etching (depth profiling) can be characterised.

Historically, a major step forward in photoelectron spectrometry was the work of Robinson and Young in 1930, who the first observed the "chemical shift". The first precision electron spectrometer was investigated by Siegbahn's group in 1950's but the first commercial XPS instrument appeared by the end of the 1960's. With the development of digital systems, multichannel detection and higher analyser transmission, the application of XPS in materials research increased dramatically around 1985 and now, this is one of the most advanced and complete modern instruments for surface investigation.

The XPS technique allows characterising different types of materials such as metals, alloys, polymers, semiconductors, geological and biological samples, and generally any surface which supports high vacuum systems. Recently, the modern electronic materials like graphene or sophisticated thin layered structures require the spe-

*corresponding author; e-mail: papis@ite.waw.pl cial approach in XPS surface measurements [1-3]. On the other hand, XPS combined with another technologies, like $\mathrm{Ar}^{+}$ion sputter etching creates a new applications of this technique. Especially, a cluster ion which contains several thousands of atoms causes unique sputtering effects for surface and can be used as a unique cleaning technique [4].

In this work, the crucial aspects of XPS such as chemical state analysis, depth profiling, mapping and thickness calculation has been presented. The metal compounds (alloys and oxides), graphene and type-II antimonides-based superlattices have been measured and examined.

\section{Experimental}

The XPS study was performed on type II InAs/GaSb superlattice structures ( $22 \mathrm{SLs}), \mathrm{Ti}_{2} \mathrm{O}_{5}$, graphene and metal alloys samples. T2Sls structures consisting of 30 period 10 monolayers (ML) InAs/9 ML GaSb were grown on undoped (100) GaSb substrates by molecular beam epitaxy. Two types of graphene samples were used in these experiments. The first was epitaxial graphene grown on a $\mathrm{SiC}$ substrate, the second graphene grown by the chemical vapour deposition (CVD) method on copper subsequently transferred onto a $\mathrm{SiO}_{2}$ substrate. The metal alloy was composed of $\mathrm{Co}, \mathrm{Al}, \mathrm{Cr}, \mathrm{Ni}$, and $\mathrm{Fe}$ metals.

Thermo Scientific K-Alpha X-ray Photoelectron Spectrometer used in these experiments is a fully integrated, monochromatic small-spot system. State-of-the-art performance, reduced cost of ownership, increased ease of use and compact size make K-Alpha system the ideal solution for many existing, as well as new surface analysis application areas. K-Alpha equipment is designed for a multiuser environment and is the first XPS tool to deliver a fully automated workflow from sample entry to report generation. 
The award-winning K-Alpha spectrometer features superior performance, fast analysis and outstanding chemical detectability. By combining cutting edge mono XPS performance with intelligent automation and intuitive control, the K-Alpha XPS is designed for a multi-user environment to meet the requirements of both XPS analysis and newcomers to the technique. Analytical options now include a tilt module for Angle Resolved XPS data collection and a recirculating inert-gas glove box for transfer of air-sensitive samples. This is an innovative system for surface characterisation of many different materials like metals, polymers, semiconductors and bio-materials. A large range of samples can be analysed: small features chemical images layer structures, designed for easy use automatic analysis of multiple samples, recipe modes for batch analysis and comprehensive software.

\section{Results}

The comparison of XPS survey spectra of $\mathrm{Ta}_{2} \mathrm{O}_{5}$ surface before and after $\mathrm{Ar}^{+}$cluster ion cleaning has been shown in Fig. 1. This result indicates that the native oxygen-based contamination was significantly reduced whereas the adventitious carbon contamination was effectively removed after $\mathrm{Ar}^{+}$cluster ion cleaning.

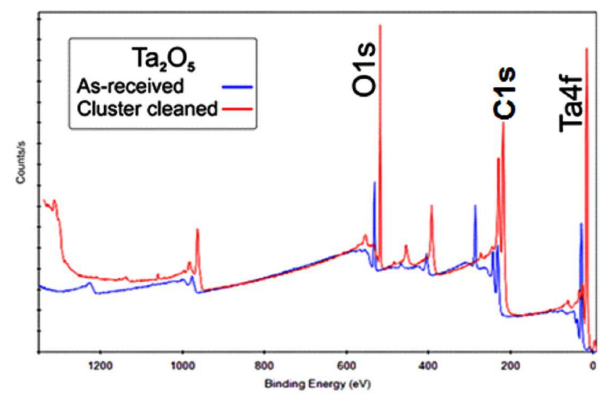

Fig. 1. Comparative survey spectra for $\mathrm{Ta}_{2} \mathrm{O}_{5}$ before and after $\mathrm{Ar}^{+}$cluster ion cleaning.

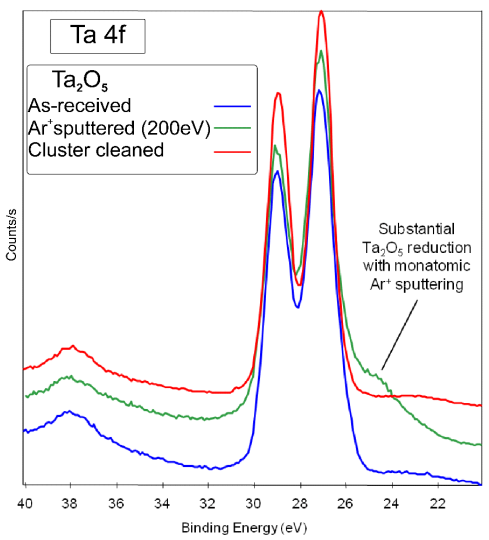

Fig. 2. Comparison of Ta $4 f$ spectrum for $\mathrm{Ta}_{2} \mathrm{O}_{5}$ before and after $\mathrm{Ar}^{+}$sputter etching and $\mathrm{Ar}^{+}$cluster ion cleaning.

Figure 2 presents the comparative Ta $4 f$ XPS spectra for $\mathrm{Ta}_{2} \mathrm{O}_{5}$ surface before cleaning (as received) and af- ter monoatomic $\mathrm{Ar}^{+}$ion sputtering and $\mathrm{Ar}^{+}$cluster ion cleaning. Low energy monoatomic $\mathrm{Ar}^{+}$ion sputtering causes a significant reduction of $\mathrm{Ta}_{2} \mathrm{O}_{5}$. This spectrum shows clear signs of reduction - the shoulder at low binding energy, which is not present in spectrum for the $\mathrm{Ar}^{+}$ cluster ion cleaned $\mathrm{Ta}_{2} \mathrm{O}_{5}$ surface. Generally, the cluster ion beams can be used to successfully clean inorganic surfaces without inducing chemical changes. The use of $\mathrm{Ar}^{+}$cluster sources has the potential to resolve XPS preferential sputtering problems for many important technological functional materials and thin films.

The results of XPS measurements of T2 InAs/GaSb are presented in Figs. 3 and 4. Analysis of Sb $3 d, \mathrm{Ga} 2 p$ and In $3 d$ indicates to presence of the native oxides layer and $\mathrm{Sb}$ and $\mathrm{In}$ metals on the superlattice surface. The depth profile (Fig. 5) shows the multilayered InAs/GaSb superlattice structure. The thickness of one pair of individual InAs and GaSb is characterized by $6.3 \mathrm{~nm}$ thickness.

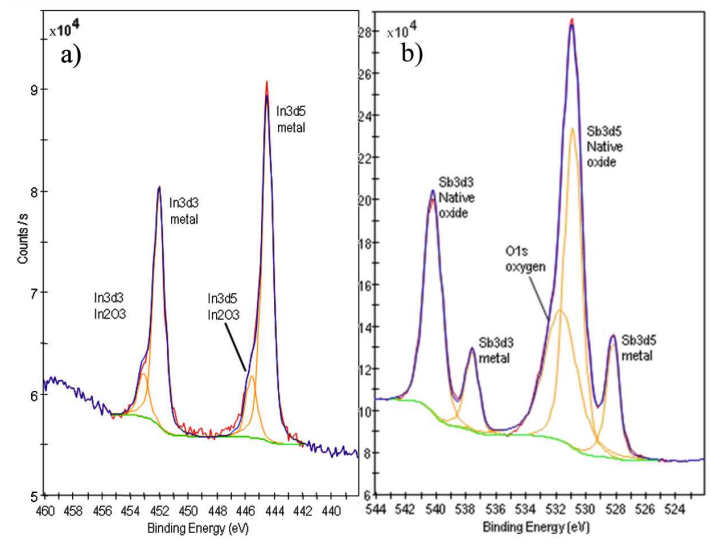

Fig. 3. XPS spectrum of: (a) In $3 d$, (b) Sb $3 d$ for T2 InAs/GaSb SLs.

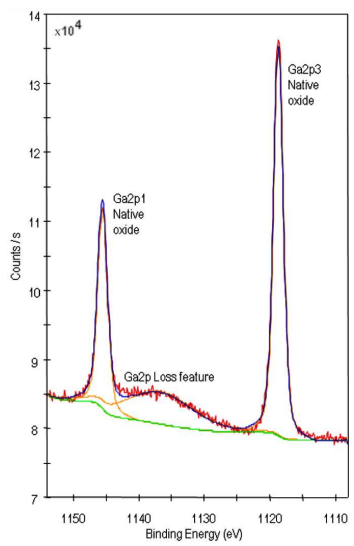

Fig. 4. XPS spectrum of Ga $2 p$ for T2 InAs/GaSb SLs.

The Thermo Scientific K-Alpha XPS spectrometer was used to analyse metal alloys samples using point analysis. In Table I a list of current elements for not cleaning (as-received) and sputtered metal alloy sample is given with 


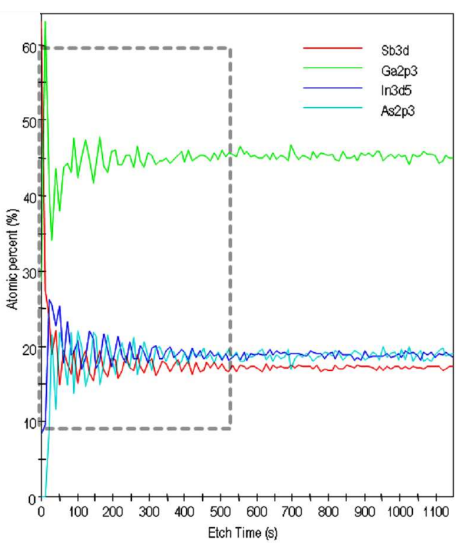

Fig. 5. The depth profile for T2 InAs/GaSb SLs.

their atomic contamination. These results show the high level of $\mathrm{C}, \mathrm{O}, \mathrm{F}, \mathrm{S}, \mathrm{N}$, and $\mathrm{Na}$ contamination in metal alloy sample. After rapid sputter cleaning, the high levels of contamination have already been greatly reduced. This allowed more accurate quantification of elemental data.

TABLE I

Comparison of metal alloy sample before and after sputter cleaning measurements.

\begin{tabular}{c|c|c}
\hline \hline \multirow{2}{*}{ Element } & \multicolumn{2}{|c}{ Atomic concentration [\%] } \\
\cline { 2 - 3 } & As-received & After sputter-cleaning \\
\hline $\mathrm{C}$ & 47.74 & - \\
$\mathrm{O}$ & 31.58 & 21.76 \\
$\mathrm{~F}$ & 6.89 & - \\
$\mathrm{Fe}$ & 4.22 & 3.24 \\
$\mathrm{Al}$ & 4.02 & 19.71 \\
$\mathrm{~N}$ & 1.53 & - \\
$\mathrm{S}$ & 1.49 & - \\
$\mathrm{Na}$ & 1.33 & - \\
$\mathrm{Co}$ & 1.20 & 20.70 \\
$\mathrm{Ni}$ & - & 16.22 \\
$\mathrm{Cr}$ & - & 18.38
\end{tabular}

Additionally, after sputter cleaning, the high resolution spectra were taken to observe the chemistry within the sample, with the possibility of obtaining more precise elemental composition from the XPS spectra (Fig. 6). The high resolution XPS spectra allow to indicate the various XPS signals and identify corresponding chemical states.

The $\mathrm{Al} 2 p$ signal (Fig. 7a) shows two states - Al metal and $\mathrm{Al}_{2} \mathrm{O}_{3}$ oxide. XPS signal of $\mathrm{Cr} 2 p$ (Fig. $7 \mathrm{~b}$ ) is characteristic for multiple split components. Using the integrated knowledge base within Thermo Fisher Scientific Avantage software, the correct fitting of the multiple split structure prevents false identification of other chemical states. Aluminium shows two clear states - metal and $\mathrm{Al}_{2} \mathrm{O}_{3}$. Chromium has many multiple-split components. Using the integrated Knowledge Base within

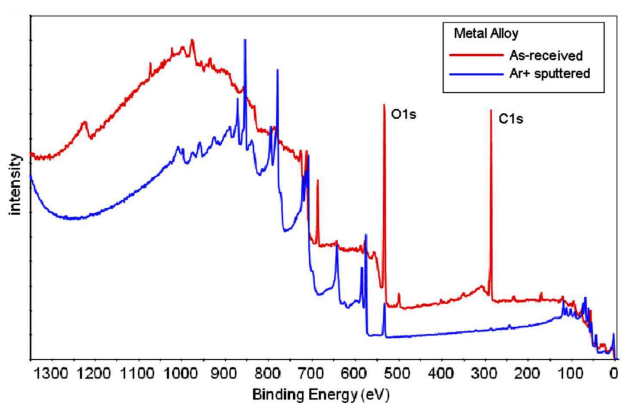

Fig. 6. Spectra for metal alloy before and after $\mathrm{Ar}^{+}$ sputter etching.

Thermo Fisher Scientific Avantage software, the correct fitting of the multiple split structure prevents false identification of other chemical states.

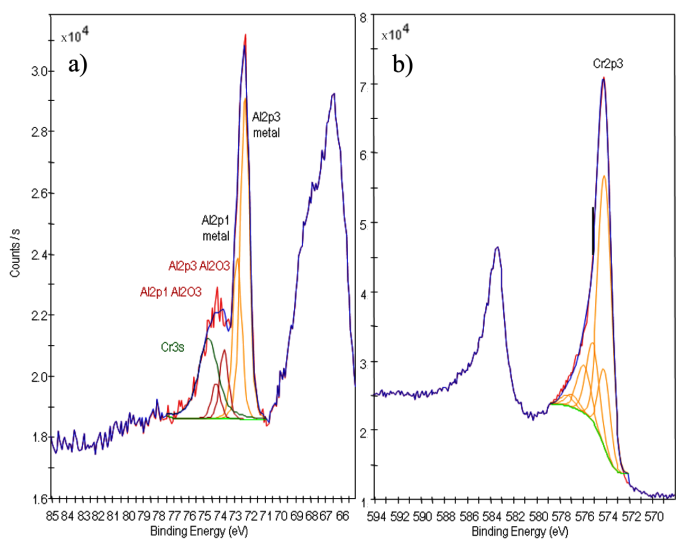

Fig. 7. XPS spectra of: (a) $\mathrm{Al} 2 p$, (b) $\mathrm{Cr} 2 p$ for metal alloy.

TABLE II

Comparison of contamination for two type graphene samples: on $\mathrm{SiC}$ and $\mathrm{SiO}_{2}$ substrates.

\begin{tabular}{c|c|c}
\hline \hline \multirow{2}{*}{ Element } & \multicolumn{2}{|c}{ Atomic concentration [\%] } \\
\cline { 2 - 3 } & Graphene on $\mathrm{SiC}$ & Graphene on $\mathrm{SiO}_{2}$ \\
\hline $\mathrm{C}$ & 64.66 & 59.77 \\
$\mathrm{O}$ & 3.41 & 23.94 \\
$\mathrm{Si}$ & 31.92 & 12.9 \\
$\mathrm{~F}$ & 0 & 2.26 \\
$\mathrm{~N}$ & 0 & 1.13
\end{tabular}

The quantification results of XPS measurements and C $1 s$ spectrum for graphene on $\mathrm{SiC}$ and $\mathrm{SiO}_{2}$ substrates are shown in Table II and Fig. 8. These results indicate the presence of $\mathrm{O}_{2}$ and $\mathrm{C}$ in both samples. For CVD graphene transferred onto the $\mathrm{SiO}_{2}$ substrate after its treatment with chemicals, $\mathrm{F}_{2}$ and $\mathrm{N}_{2}$ are also visible. However, annealing of samples is necessary to eliminate any molecules that were physisorbed on the graphene layer from the air. Additionally, the XPS spectrum for epitaxial graphene allows the analysis of both $\mathrm{C}-\mathrm{C}$ and $\mathrm{Si}-\mathrm{C}$ bonds. 


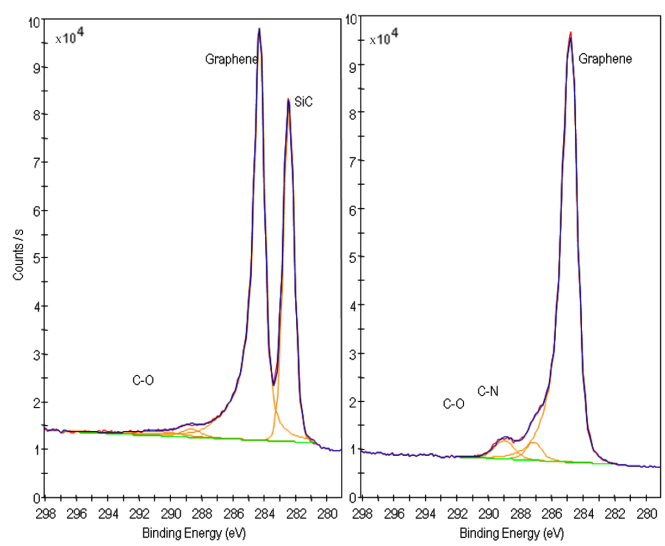

Fig. 8. XPS spectra of graphene on: (left) $\mathrm{SiC}$, (right) $\mathrm{SiO}_{2}$ substrates.

The thickness of graphene can be determined using the thickness calculator system within the Avantage software. For this calculation $3.2 \mathrm{~g} / \mathrm{cm}^{3}$ density taken from scientific reports was used.

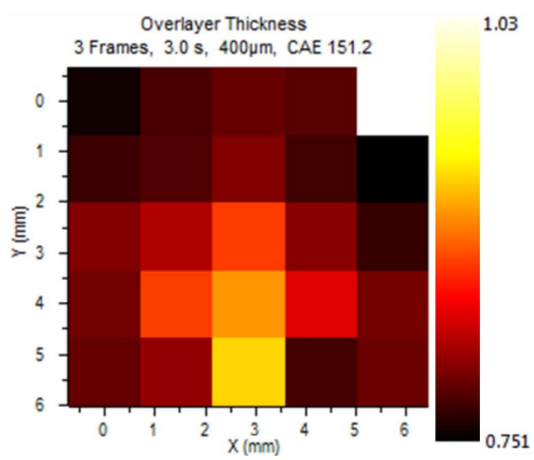

Fig. 9. 2D map of the graphene on $\mathrm{SiC}$ substrates.

TABLE III

Average thickness of graphene layer determined using the thickness calculator within the Avantage software.

\begin{tabular}{c|c}
\hline \hline Average thickness [nm] & 0.81307316 \\
Maximum thickness [nm] & 1.02921 \\
Minimum thickness [nm] & 0.751483 \\
Average thickness, excluding & 0.806353739 \\
maximum and minimum [nm] &
\end{tabular}

A 2D map of the graphene on $\mathrm{SiC}$ substrate was created (Fig. 9) and an average thickness of graphene layer was determined using the thickness calculator within the Avantage software (Table III). Using the K-Alpha 128 channel detector, multichannel snapshot mode was used for rapid data acquisition during mapping measurement. Snapshot mode allows reducing acquisition time. Consequently, at each point of this map, spectra were acquired for only $3 \mathrm{~s}$. The top of right area of this map shows the thickest layer of graphene. This method is a good way to determine of uniformity of graphene thickness. However, a 2D map would provide fuller information about the uniformity of thickness if diameter of X-ray beam was smaller. Using principal component analysis (PCA) within the Avantage data system, two components were found.

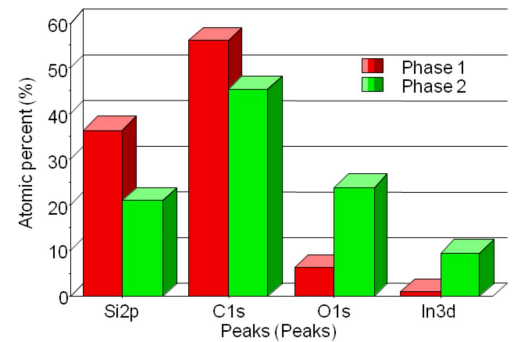

Fig. 10. Atomic concentration over the area of the graphene on $\mathrm{SiC}$ substrates.

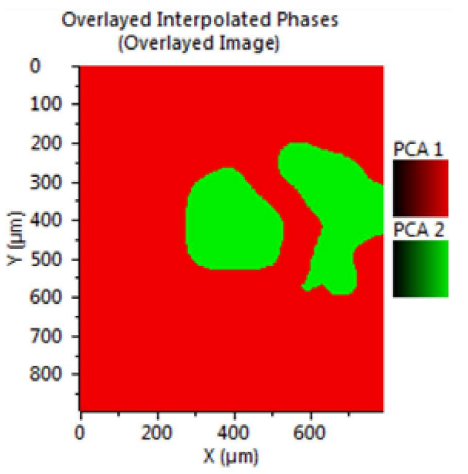

Fig. 11. Image of graphene on $\mathrm{SiC}$ substrates.

Graphs presented in Figs. 10 and 11 indicate the average atomic percentage within these components and location of the main elements on the graphene sample.

\section{Summary}

The metal alloys, $\mathrm{Ti}_{2} \mathrm{O}_{5}$, graphene and type-II InAs/ GaSb superlattice structures have been measured by using the new Thermo Scientific K-Alpha X-ray Photoelectron Spectrometer. The various measurements possibilities of XPS methods, such as chemical state analysis, depth profiling, mapping, and thickness calculation have been presented.

\section{References}

[1] E. Papis-Polakowska, J. Kaniewski, J. Szade, W. Rzodkiewicz, A. Jasik, K. Reginski, A. Wawro, Thin Solid Films 522, 223 (2012).

[2] W. Strupinski, K. Grodecki, A. Wysmolek, R. Stepniewski, T. Szkopek, P.E. Gaskell, A. Grüneis, D. Haberer, R. Bozek, J. Krupka, J.M. Baranowski, Nano Lett. 11, 1786 (2011).

[3] D. Ferrah, J. Penuelas, C. Bottela, G. Grenet, A. Ouerghi, Surf. Sci. 615, 47 (2013).

[4] H. Biederman, D. Slavinska, H. Boldyreva, H. Lehmberg, G. Takaoka, J. Matsuo, H. Kinpara, J. Zemek, J. Vac. Sci. Technol. B 19, 2050 (2001). 